\title{
Automatic and intentional memory processes in visual search
}

\author{
WALTER R. BOOT \\ University of Illinois at Urbana-Champaign, Urbana, Illinois \\ JASON S. MCCARLEY \\ Mississippi State University, Mississippi State, Mississippi \\ ARTHUR F. KRAMER \\ University of Illinois at Urbana-Champaign, Urbana, Illinois \\ and \\ MATTHEW S. PETERSON \\ George Mason University, Fairfax, Virginia
}

\begin{abstract}
Previous research has indicated that saccade target selection during visual search is influenced by scanning history. Already inspected items are less likely to be chosen as saccade targets as long as the number intervening saccades is small. Here, we adapted Jacoby's (1991) process dissociation procedure to assess the role of intentional and automatic processes in saccade target selection. Results indicate a large automatic component biasing participants to move their eyes to unexamined locations. However, an intentional component allowed participants to both reinspect old items and aid their selection of new items. A second experiment examined inhibition of return (IOR) as a candidate for the observed automatic component. IOR was found for items that had been previously examined. It is concluded that both automatic and intentional memory traces are available to guide the eyes during search.
\end{abstract}

All else being equal, search for a target among distractors would be most efficient if examined and rejected nontarget objects were never reexamined. Efficient visual search would therefore seem to depend on memory mechanisms to prevent costly revisits of attention to previously inspected items. Consistent with this speculation, data indicate that oculomotor search is generally guided away from items that have been inspected and recognized as nontargets (Gilchrist \& Harvey, 2000; Peterson, Kramer, Wang, Irwin, \& McCarley, 2001). For example, using a dynamic visual search display that prevented observers from invoking mnemonic scanning strategies, McCarley, Wang, Kramer, Irwin, and Peterson (2003) found evidence of memory for previously fixated items when fewer than four or five saccades occurred after an item had first been fixated. These findings indicate that memory for where the eyes have been does serve to guide saccade targeting.

Additional evidence for memory-guided search comes from the finding of inhibition of return (IOR) in both overt (Klein \& MacInnes, 1999) and covert (e.g., Klein, 1988; Takeda \& Yagi, 2000) search paradigms. Inhibition can be observed in these tasks through longer response times when a probe occurs at the location of a previously at-

Correspondence should be addressed to A. F. Kramer, Beckman Institute, University of Illinois at Urbana-Champaign, 405 N. Mathews, Urbana, IL 61801 (e-mail: akramer@s.psych.uiuc.edu). tended distractor. This form of memory has been described as a "foraging facilitator" (Klein \& MacInnes, 1999) since it biases the deployment of attention toward new objects and away from already inspected items, increasing the probability of finding the target of search. IOR is likely to be one form of memory used to guide attention during search, among others.

The assertion that visual search will be most adaptive if observers do not "waste" attentional fixations (overt or covert) revisiting items, however, will be true only if the benefits to search efficiency gained from short-term attention-guiding memory outweigh the costs of implementing and utilizing that memory. In fact, the costs of memory-guiding search may be substantial. Working memory is generally considered be effortful, or resource demanding, and thus might not be invoked by default as an aid to search. Wolfe, Alvarez, and Horowitz (2000), moreover, have suggested that conscious top-down processes in the control of search operate more slowly than bottom-up, "anarchic" processes. If this is the case, then intentional efforts to guide search based on memory for scan history might actually hinder overall performance.

A solution to these difficulties might be to rely on implicit, automatic memory processes to guide attention during search rather than explicit, intentional processes. Various forms of implicit memory are already known to moderate search performance. Both long-term contextual cuing (Chun \& Jiang, 1998) and short-term priming-of- 
popout (Maljkovic \& Nakayama, 1994), for example, appear to guide attention implicitly and automatically. Operating without conscious effort, such processes can facilitate search while leaving conscious, effortful processes free to support more resource-intensive mental activities (Chun \& Nakayama, 2000). Furthermore, they can circumvent the "volitional bottleneck" that makes intentional control of attention inefficient (Wolfe et al., 2000).

All of this suggests that the role of memory for search history in guiding visual search may be largely automatic, subject to little intentional control. We tested this speculation here using an adaptation of Jacoby's (1991) process dissociation procedure (PDP) in conjunction with a variant of the gaze-contingent saccade targeting task of McCarley et al. (2003). Each trial comprised a series of forced-choice events in which participants selected between a saccade target item that had previously been fixated and one that had not been fixated. Three experimental conditions were compared. In one, the go-toold condition, participants were instructed to saccade toward items that had been previously fixated. In another, the go-to-new condition, they were instructed to saccade toward items that had not already been fixated. If memory processes that underlie saccade targeting are purely intentional, participants should do equally well in these conditions. However, if automatic memory mechanisms work to bias saccades away from previously visited items, participants should perform better in the go-to-new than the go-to-old condition. Additionally, a go-to-either condition was included to assess the relative contribution of automatic processes during free search. If saccade targeting is automatic, then participants should be biased to saccade to the new item even when given no instructions to do so. That is, memory performance should be similar in the go-to-new and go-to-either conditions.

The gaze-contingent saccade targeting paradigm of McCarley et al. (2003) was chosen because it isolates memory for previously fixated items by disallowing mnemonic strategies such as stereotyped scanning or geographic chunking of locations. Additionally, by enabling memory performance to be assessed as a function of the lag since a given item target has been fixated, it provides a straightforward measure of functional memory capacity. The modified paradigm employed here differs from typical search in that participants move their eyes specifically on the basis of instruction rather than in an attempt to find a particular target item among distractors. However, this represents a way to determine the memory mechanisms available during search since resources are primarily focused on using memory to guide saccade target selection. Of course, as indicated above, our paradigm also differs from typical search in that our stimuli are presented in a gaze-contingent fashion. However, given the similar estimate of memory span in our paradigm and that obtained by Gilchrist and Harvey (2000) in a more traditional search paradigm, the gazecontingent nature of our task does not appear to distort the memory processes that underlie search.
To preview the results, in Experiment 1, the memory processes that guide saccade target selection were found to be largely automatic. Performance in the go-to-new condition and go-to-either condition was similar. Additionally, performance in the go-to-old condition was poorer than in the go-to-new condition, suggesting that the automatic bias is difficult to overcome. Experiment 2 suggests that the mechanisms that underlie IOR subserve the automatic component of saccade target selection.

\section{EXPERIMENT 1}

The PDP was used to examine the role of automatic and intentional memory processes in the guidance of eye movements. Participants were asked to make a series of eye movements in a gaze-contingent task like that of McCarley et al. (2003). Participants were instructed to intentionally direct their eyes to items on the basis of scan history or to move their eyes regardless of this history. If the memory processes that guide the eyes during search are largely intentional, participants should perform equally well in the go-to-old and go-to-new conditions. In other words, the refixation frequency in the go-to-old condition should be equal to one minus the refixation frequency in the go-to-new condition. Conversely, if these memory processes are largely automatic, participants should be better at selecting new items than old items, and should be biased to move their eyes to new items even without instructions to do so.

\section{Method}

Participants. Thirteen participants with normal vision (5 males) ranging in age from 18 to 28 years were paid for their participation.

Apparatus. A Pentium-based computer with a 21-in. SVGA color monitor was used to present all stimuli. Eye movements were recorded with an Eyelink eye tracker. A chinrest positioned $87 \mathrm{~cm}$ from the screen was used to stabilize head position. An eye movement was classified as a saccade if its distance exceeded $.2^{\circ}$ and either its acceleration reached $9,500 \% \mathrm{sec}^{2}$ or its velocity reached $30 \% \mathrm{sec}$.

Stimuli. Participants viewed dynamic displays containing gray crosses $\left(.33^{\circ} \times .33^{\circ}, 11.35 \mathrm{~cd} / \mathrm{m}^{2}\right)$ on a black background $\left(.14 \mathrm{~cd} / \mathrm{m}^{2}\right)$. With the exception of the instructions read to participants, all experimental conditions were identical. Trials were divided into discrete events in which one, two, or three crosses appeared on screen (Figure 1). To build a history of old items (previously fixated items), participants were first presented with a series of four crosses that they were required to fixate. A trial started with the participant gazing at a white fixation point and pressing the space bar. As soon as this occurred, one gray cross appeared, which subjects were required to fixate (Event 1). During this eye movement, a second item was added to the display (Event 2). Participants were then required to fixate this item. As participants moved their eyes to this item, the previously fixated item was removed and another item to saccade to was added to the display (Event 3 ). This continued until participants had fixated four objects. As participants were making this eye movement to the fourth item, one item was randomly chosen from the list of previously fixated items and another new item equidistant to this item appeared in the display (Event 5). At this point, participants had two potential saccade targets. One item was an old item that had already been fixated at some point in the trial, and one item was new. If the old item chosen to be presented was the item just previously fixated, this item remained persistent in the dis- 


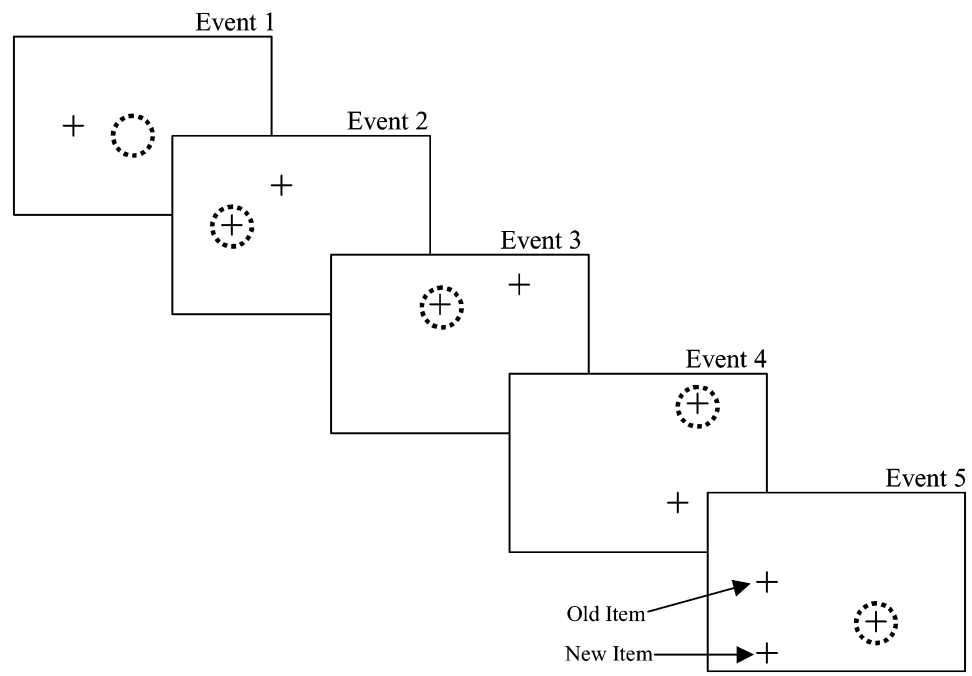

Figure 1. An example of the sequence of events for a trial in Experiment 1. The dotted circle represents current gaze position. Participants were presented with a series of four saccade targets (Events 1-4) to build a history of previously visited items. Starting at the fifth event, the participants were asked to saccade to the new item (an item that had not been fixated previously), the old item (an item that had been fixated at some point in the trial), or either item.

play. If the old item chosen was not the item previously fixated, the previously fixated item was removed from the display and the old item was redrawn in the location in which it had appeared previously. From this point on in the trial, with each saccade a participant made, one randomly chosen old item was presented as well as a new item equidistant from the about-to-be-fixated item and the old item. ${ }^{1}$

Stimulus placement was constrained so that stimuli were placed more than $4.31^{\circ}$ and less than $8.69^{\circ}$ from the currently fixated item. Additionally, old and new items appeared at least $4.31^{\circ}$ apart. If no suitable stimulus locations could be found, the trial ended. Otherwise, the trial ended after 11 saccades had been made. A saccade was recognized as going to a particular item when the eyes crossed an imaginary bounding circle with a diameter of $1.74^{\circ}$.

Procedure. Participants completed one practice session and two experimental sessions on separate days with three blocks (go-toold, go-to-new, go-to-either) per session. Blocks were completed in the same order each day and participants were randomly assigned different block orders. In all conditions, participants were told to move their eyes to the first four stimuli appearing in the display, and that after the fourth saccade they would be presented with a choice of two saccade targets. In the go-to-new condition, participants were told that when this happened they should move their eyes to the object that they had not fixated previously. Participants were told that for the remainder of the trial, they should always try to move their eyes to stimuli they had not already looked at. In the go-to-old condition, participants were asked to move their eyes to objects that they had fixated already. In the go-to-either condition, participants were told that they should move their eyes to whichever item they preferred, without concern for previous fixations. On each day, participants completed three blocks of 60 trials each.

\section{Results and Discussion}

The first session was considered practice. Refixation analyses included only data from the first choice point within each trial (i.e., the first opportunity in which participants had the option to saccade to either an old item or a new item) since after this point, trial history diverged depending on the particular condition.

Refixation analysis. Figure 2 depicts revisitation data for each instruction condition. Data were submitted to a two-way repeated measures analysis of variance (ANOVA) with instruction (go-to-old, go-to-either, go-to-new) and lag $(1,2,3)$ as factors. Consistent with previous findings, a reliable main effect of lag was observed $[F(2,24)=$ $54.28, p<.001$ ], indicating a declining memory for search history. Of more immediate importance, a reliable main effect of instruction was observed $[F(2,24)=10.27$, $p<.01]$. The interaction of lag $\times$ instruction was not significant $[F(2,24)=1.31, p=.28]$. Data for each condition were collapsed across values of lag and submitted to a series of planned comparisons via paired and onesample $t$ tests. Results demonstrate the operation of both intentional and automatic processes. Refixation rates in the go-to-old condition were significantly higher than in the go-to-new condition $[t(12)=3.37, p<.01]$. Similarly, refixation rates in the go-to-either condition were reliably lower than in the go-to-old condition $[t(12)=$ $3.12, p<.01]$, but reliably higher than in the go-to-new condition $[t(12)=2.27, p<.05]$. Observers thus were clearly capable of exercising intentional memory processes to control saccade target selection. Nonetheless, observers' ability to conform to instructions in the go-toold condition was poorer than predicted by a purely intentional model of memory. Refixation rate in the go-toold condition, that is, was substantially less than the value of one minus refixation rate in the go-to-new condition $[t(12)=8.44, p<.001]$. Indeed, refixation rates in go-to-old condition for the first lag even fell short of 


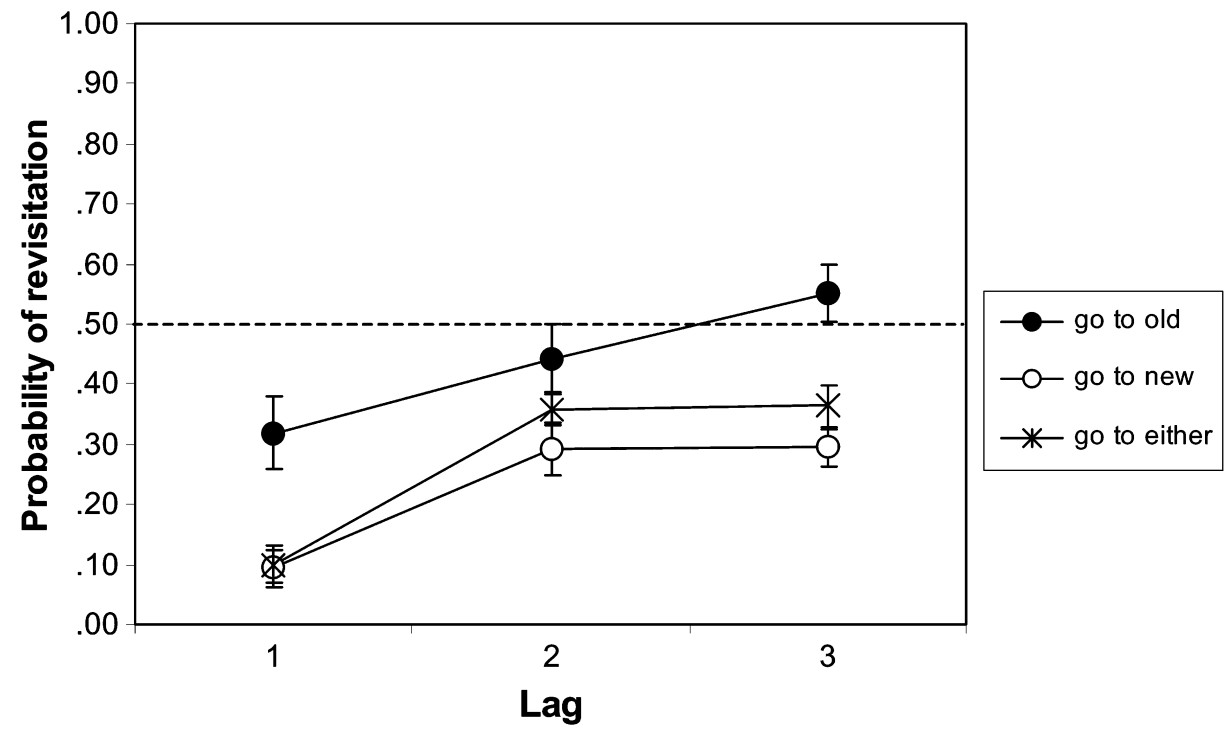

Figure 2. Proportion of revisitations in the go-to-new, go-to-old, and go-to-either conditions for the first choice point.

chance level $[t(12)=2.94, p<.05]$. Thus, the memory processes guiding saccade targeting away from previously visited items could not be fully overcome by intentional processes. Consistent with this conclusion, the refixation rate in the go-to-either condition was only modestly higher than in the go-to-new condition, but was substantially lower than in the go-to-old condition. ${ }^{2}$

Saccadic latency analysis. Saccade latencies were examined for converging evidence of the distinction between automatic and intentional processes in saccade target selection. Note that the observers' task load in the go-to-new and go-to-old conditions was nominally the same; both sets of instructions asked observers to keep track of and make decisions based on their saccade history. Under go-to-new conditions, however, intentional processes would have worked in concert with automatic processes. Under go-to-old instructions, intentional processes would have had to overcome automatic processes. This suggests that the time necessary for saccade initiation should have been longer under go-to-old than go-tonew conditions. To the extent that saccade targeting under the go-to-new condition was guided by automatic processes, go-to-either saccade latencies should have been similar to go-to-new latencies, and faster than go-to-old latencies. Planned comparisons confirmed these predictions. Mean saccade latency with go-to-old instructions $(M=436 \mathrm{msec})$ was significantly longer than with either go-to-new instructions $[M=403 \mathrm{msec}, t(12)=3.45$, $p<.01]$ or go-to-either instructions $[M=382 \mathrm{msec}$, $t(12)=2.40, p<.05]$. Latencies for the go-to-new condition and the go-to-either condition were not reliably different $[t(12)=1.00, p=.34] .{ }^{3}$ The revisitation data and the latency analysis would suggest that saccade tar- geting is relatively automatic but that intention can improve saccade targeting.

\section{EXPERIMENT 2}

Experiment 2 was conducted to examine the mechanisms underlying the results of Experiment 1, specifically the strong bias to saccade away from old items. Latencies were longer in the go-to-old condition than in the go-to-either condition, but latencies did not differ significantly between the go-to-either and go-to-new conditions. If latency differences were due to an intentional component, longer latencies would be expected in both the go-to-old and go-to-new conditions than in the go-toeither condition. However, longer latencies only in the goto-old condition would suggest that latency differences are due to having to overcome an automatic bias that drives the eyes away from previously fixated items. Many studies have demonstrated IOR (see Klein, 2000, for review). It is conceivable that the mechanism(s) responsible for IOR might be the automatic component observed in Experiment 1. Experiment 2 used a more traditional IOR paradigm that prevented any decision-making component from influencing saccadic latency. This paradigm combined the dynamic visual search task of McCarley et al. (2003) with a speeded saccadic probe task similar to that of Klein and MacInnes (1999). Participants were asked to make a speeded eye movement to a probe as soon as they detected it. IOR effects were measured in terms of saccadic latencies of the eye movements going to the probed item. If the processes that produce IOR serve as the automatic memory component of saccade target selection observed in the previous experiment, 
participants should be slower to initiate a saccade to previously fixated items than to new items.

\section{Method}

Participants. Ten participants with normal vision (5 males) ranging in age from 21 to 26 years were paid for their participation.

Apparatus. The same apparatus was used as in the previous experiment.

Stimuli. Participants viewed dynamic displays similar in nature to those of Experiment 1. These displays contained gray rotated
L-shaped items $\left(.14^{\circ} \times .14^{\circ}, 11.35 \mathrm{~cd} / \mathrm{m}^{2}\right)$ on a black background $\left(.14 \mathrm{~cd} / \mathrm{m}^{2}\right)$. These L-shapes could be rotated $0^{\circ}, 90^{\circ}, 180^{\circ}$, or $270^{\circ}$ from upright. During a trial, a red box $\left(.43^{\circ} \times .43^{\circ}, 18.93 \mathrm{~cd} / \mathrm{m}^{2}\right)$ appeared around one item in the display and participants were to fixate this item as soon as they detected it.

Procedure. The procedure was similar to that in the go-to-either condition of Experiment 1. The observers' task was to move their eyes to items that appeared in the display until the probe was presented, and then to make a speeded saccade to the item within the red box. As in Experiment 1, participants were first presented with a series of saccade targets to build a history of previously fixated

\section{Old Probe: Persistent}

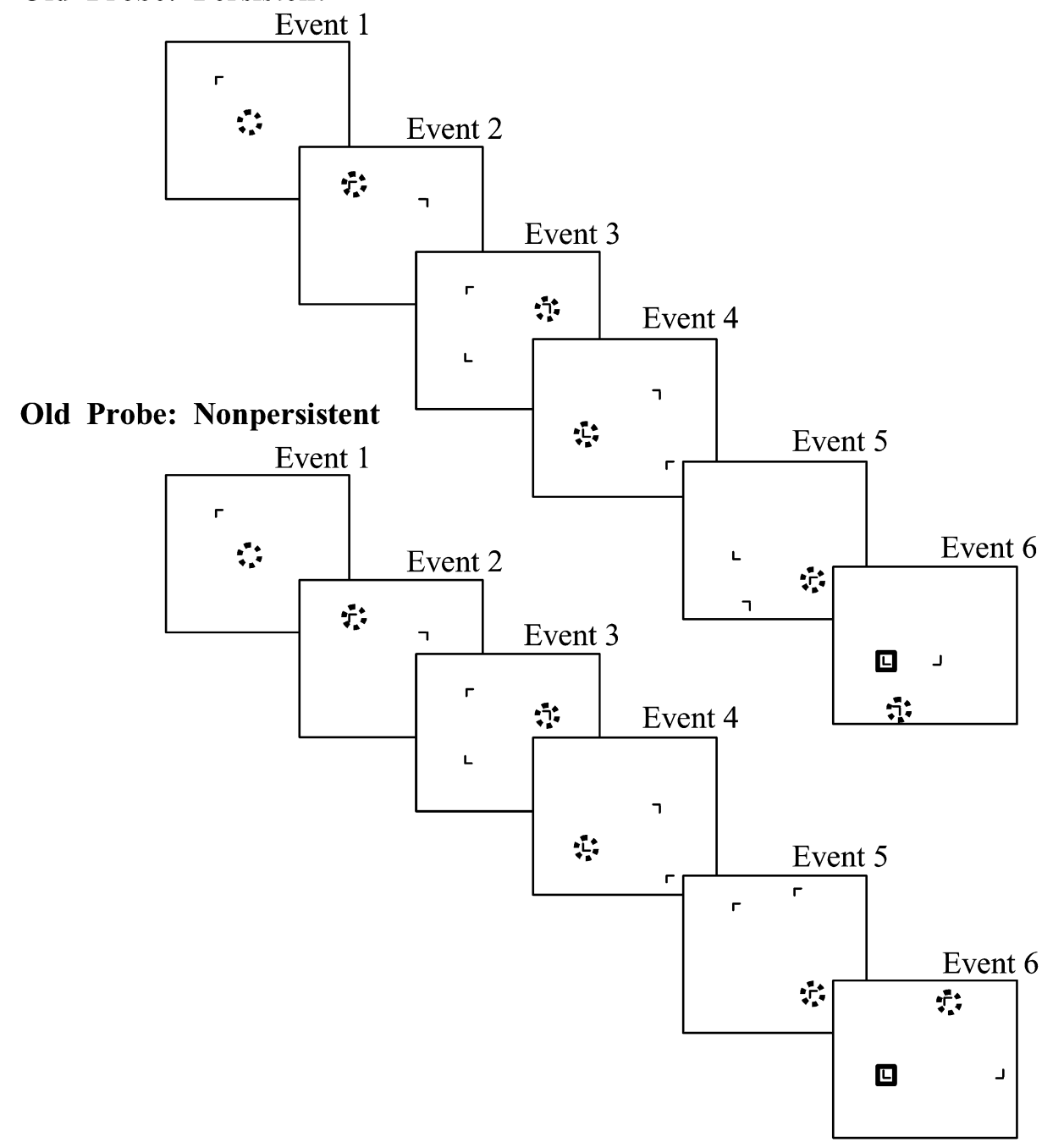

Figure 3. Example sequence of events for two trials in Experiment 2. The dotted circle represents current gaze position. Participants were required to make a series of eye movements to stimuli appearing on screen. When presented with a choice of items, beginning at Event 3, the participants were asked to saccade to whichever item they preferred. The participants were allowed to choose to saccade to any item up until the presentation of the probe. When the probe occurred, the participants were asked to make a speeded saccade to the probed item. Either the item visited on the third saccade (Event 4) or an item equidistant from this item was probed two saccades later (Event 6). When the probed item was a previously visited item, it could be either persistent (remaining on screen during the intervening saccade) or nonpersistent (disappearing after it has been visited and reappearing at the time of the probe). The figure illustrates both a persistent old item probe and a nonpersistent old item probe. 
items (Figure 3). Experiment 2 differed from Experiment 1 in the number of initial items presented to build a history of old items before participants were allowed a choice of saccade targets (two items in the present experiment as opposed to four items in the previous experiment). Otherwise, stimulus presentation was identical. During the fifth saccade, as in prior events, a previously fixated item was presented, as well as a new item equidistant from this previously fixated item and the about-to-be-fixated item. The previously fixated item in this case was not determined by chance, but was specifically chosen to be the item fixated two fixations back. In addition to these two items, the red probe was drawn either around the previously fixated item or around the new item. When the probe was drawn around an old item, this item could either be persistent (remaining on screen during the intervening saccades between when it was visited and probed) or nonpersistent (being removed after it has been visited and appearing again at the time of the probe). Participants were instructed to fixate the probed item as quickly as possible when they detected it. Latency of the saccade going to the probed item was used to measure IOR. The probe was visible for $1 \mathrm{sec}$ before the trial ended. Each participant completed one block of 300 trials. The probe could occur equally often around an old persistent item, an old nonpersistent item, or a new item.

\section{Results and Discussion}

Discarded data. On $19 \%$ of trials, stimulus placement restrictions caused the trial to end before the probe could be drawn. If probe presentation did not occur while the eyes were in flight (i.e., during the saccade occurring between Events 5 and 6), these trials were discarded to eliminate any effect this transient might have had on saccadic reaction time $(30 \%)$. Trials were also discarded if the first saccade after the probe was drawn did not land within $1.74^{\circ}$ of the probe location (25\%). Finally, an outlier removal process removed all latencies either above or below $2 S D$ of the mean of each condition, resulting in the additional loss of $5 \%$ of remaining trials. This left on average 120 saccades per observer. ${ }^{4}$

Saccadic latency analysis. Excluding the probe event, memory performance and average saccadic latency data were similar to performance in the go-to-either condition of Experiment 1. Of primary interest was the latency of the critical saccade to the probe location. A one-way repeated measures ANOVA with probe type as the factor (old persistent, old nonpersistent, control) revealed a reliable effect $[F(2,18)=12.32, p<.001]$. Paired $t$ tests were used to further explore the effect of probe type. These $t$ tests revealed a significant slowing of saccades both to old persistent items $[M=229 \mathrm{msec}, t(9)=6.42, p<.001]$ and to old nonpersistent items $[M=224 \mathrm{msec}, t(9)=$ $3.24, p<.05]$ relative to saccades to the new item $(M=$ $202 \mathrm{msec}$ ). A comparison between old persistent items and old nonpersistent items revealed no significant difference $[t(9)=.74, p=.48]$.

These results confirm that IOR operates in the present paradigm. Interestingly, IOR was observed for both persistent and nonpersistent objects. At first this might appear inconsistent with the previous research finding that the removal of the search display removes inhibitory tagging (e.g., Klein \& MacInnes, 1999). However, Tipper, Jordan, and Weaver (1999) have suggested that in displays containing separate objects related to one another in terms of spatial location (such as the sparse displays used here), location-based tagging might be used, but when the display is given more context and objects in the scene are more integrated (as is the case in more natural scenes), tagging might be primarily object based.

\section{GENERAL DISCUSSION}

Search would be most efficient if past history were taken into account in the guidance of attention. Currently there exists evidence that memory mechanisms operate during overt search (Gilchrist \& Harvey, 2000; McCarley et al., 2003; Peterson et al., 2001). The present results suggest that the memory mechanisms guiding the eyes away from recently fixated items during search are predominately automatic. By default, participants are more likely to direct their eyes to new items even when given no instruction to do so. An automatic memory mechanism appears to bias participants to move their eyes away from previously inspected locations. IOR, which has been described as a "foraging facilitator" (Klein \& MacInnes, 1999), appears to serve this function. Inhibition of previously inspected items was observed in the form of longer latencies of saccades to old items relative to previously uninspected items. Interestingly, not only did this inhibition appear to encourage participants to select the new item more often, but it also interfered with participants' ability to saccade to the old item when participants were instructed to do so in Experiment 1. This inhibition appears to decline over time. With increasing lag, the saccadic reaction time difference between when the eyes go to the new item relative to when the eyes go to the old item decreases, corresponding to the decline in memory performance (Figure 4).

Intentional memory processes also appear to be available during search. Intention enabled participants to select new objects better than when they were given no instruction. These memory processes also allowed participants to select items that they had previously inspected as saccade targets. One form of memory that might provide some benefit in saccade target selection, especially for Lag 1 items, is iconic memory. However, it is uncertain how useful such representations might be since iconic memory for location information does not appear to be robust across eye movements (Irwin, 1992). Instead, we believe that the intentional component serving saccade target selection is more likely to make use of visual short-term or transsaccadic memory (Irwin, 1996; Luck \& Vogel, 1997). The results presented here suggest that intentional processes do not improve performance to a great extent. Although both memory mechanisms appear to be available during search, participants might rely more on automatic processes given that there is only a minor benefit of using a more effortful, intentional strategy in selecting saccade targets. Interestingly, Woodman, Vogel, and Luck (2001) found no decrease in search efficiency when working memory was full. A working memory load would be expected to interfere with intentional, but not automatic, memory 

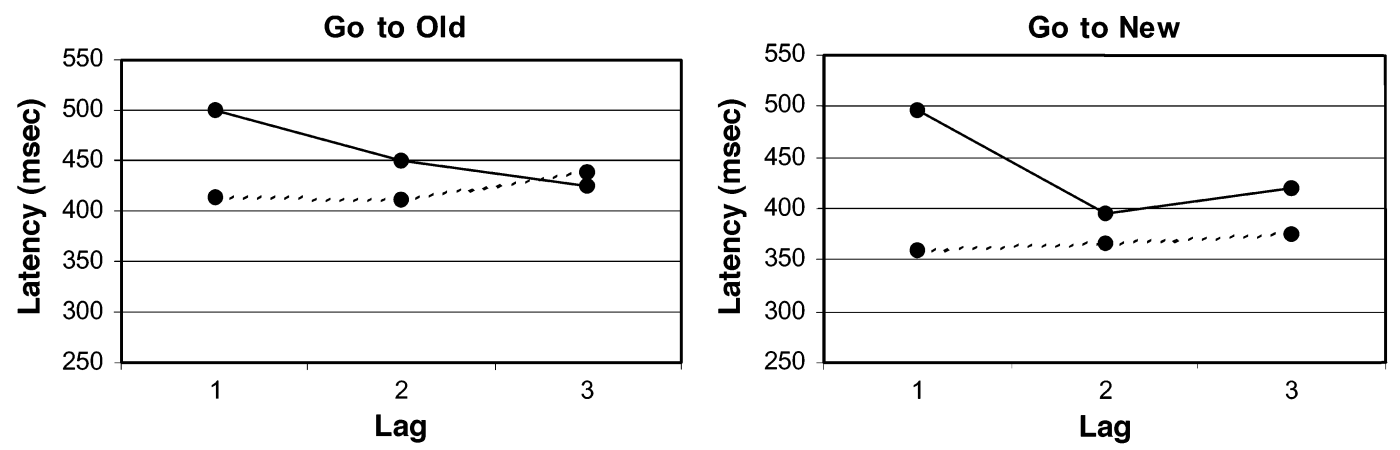

Go to Either
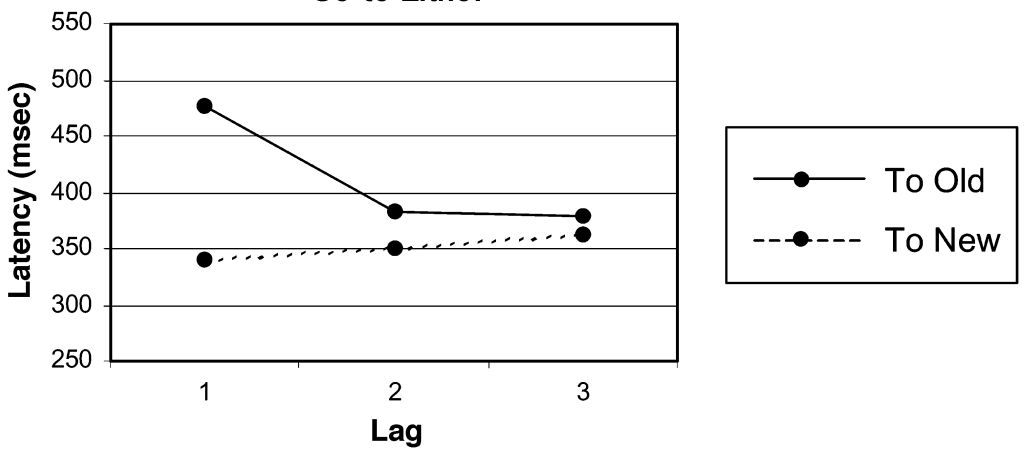

Figure 4. Latency data for each instruction condition in Experiment 1 conditional upon whether the eyes go to the old item or the new item.

processes. Other results cast doubt upon whether intentional memory for previously examined locations can be used to guide attention effectively during oculomotor search. Pearson and Sahraie (2003) found that the act of programming and executing saccadic eye movements disrupted visuospatial working memory, the memory mechanism that presumably would allow participants to intentionally guide saccades on the basis of remembered search locations. These results are consistent with our finding that the control of attention shifts during search is largely unconscious.

\section{REFERENCES}

Chun, M. M., \& Jiang, Y. (1998). Contextual cueing: Implicit learning and memory of visual context guides spatial attention. Cognitive Psychology, 36, 28-71.

CHUN, M. M., \& NAKAYAMA, K. (2000). On the functional role of implicit visual memory for the adaptive deployment of attention across scenes. Visual Cognition, 7, 65-81.

Gilchrist, I. D., \& HARVEY, M. (2000). Refixation frequency and memory mechanisms in visual search. Current Biology, 19, 1209-1212.

IRWIN, D. E. (1992). Memory for position and identity across eye movements. Journal of Experimental Psychology: Learning, Memory, \& Cognition, 18, 307-317.

IRWIN, D. E. (1996). Integrating information across saccadic eye movements. Current Directions in Psychological Sciences, 5, 94-100.

JACOBY, L. L. (1991). A process dissociation framework: Separating automatic from intentional uses of memory. Journal of Memory \& Language, 30, 513-541.

KLEIN, R. M. (1988). Inhibitory tagging system facilitates visual search. Nature, 334, 430-431.
KleIn, R. M. (2000). Inhibition of return. Trends in Cognitive Sciences, 4, 138-147.

KLEIN, R. M., \& MacInNES, W. J. (1999). Inhibition of return is a foraging facilitator in visual search. Psychological Science, 10, 346-352.

LuCK, S. J., \& Vogel, E. K. (1997). The capacity of visual working memory for features and conjunctions. Nature, 390, 279-281.

MacInNES, W. J., \& KLEIN, R. M. (2003). Inhibition of return biases orienting during the search of complex scenes. Scientific World Journal, 3, 75-86.

MalJKovic, V., \& NaKayama, K. (1994). Priming of pop-out: I. Role of features. Memory \& Cognition, 22, 657-672.

McCarley, J. S., Wang, R. F., Kramer, A. F., Irwin, D. E., \& PeterSON, M. S. (2003). How much memory does oculomotor search have? Psychological Science, 14, 422-426.

PeArson, D. [G.], \& Sahraie, A. (2003). Oculomotor control and the maintenance of spatially and temporally distributed events in visuospatial working memory. Quarterly Journal of Experimental Psychology, 56A, 1089-1111.

Peterson, M. S., Kramer, A. F., Wang, R. F., Irwin, D. E., \& McCarley, J. S. (2001). Visual search has memory. Psychological Science, 12, 287-292.

TAKEDA, Y., \& YAGI, A. (2000). Inhibitory tagging in visual search can be found if search stimuli remain visible. Perception \& Psychophysics, 62, 927-934.

TiPPER, S. P., JORDAN, H., \& WEAVER, B. (1999). Scene-based and objectcentered inhibition of return: Evidence for dual orienting mechanisms. Perception \& Psychophysics, 61, 50-60.

Wolfe, J. M., Alvarez, G. A., \& Horowitz, T. S. (2000). Attention is fast but volition is slow. Nature, 406, 691.

Woodman, G. F., Vogel, E. K., \& LUCK, S. J. (2001). Visual search remains efficient when visual working memory is full. Psychological Science, 12, 219-224.

YANTIS, S., \& JONIDES, J. (1984). Abrupt visual onsets and selective attention: Evidence from visual search. Journal of Experimental Psychology: Human Perception \& Performance, 10, 601-621. 


\section{NOTES}

1. One concern might be that attention capture by abrupt onsets could influence memory results, especially given that on some events the old item remained persistent in the display while the new item appeared as an onset (Yantis \& Jonides, 1984). However, given that stimulus presentation occurred while the eyes were in flight, saccadic suppression should greatly weaken or eliminate the perception of onset transients, and thus this concern should be minimized.

2. It might be argued that the first four items presented on screen could have biased participants to saccade to new items since these items are necessarily new. However, analyses of all choice points, in addition to only choice points occurring late in the trial, were consistent with the reported analyses, making it unlikely that this was the source of the observed bias. Data from the first choice have been reported given that trial history differed after this point. In particular, the memory set for old items (the number of possible old items available to present during the trial) was smaller in the go-to-old condition if participants were able to successfully direct their eyes to old items during the trial.

3. Analysis of only latencies of saccades at the first choice point indicated identical effects, although the overall ANOVA did not reach significance $[F(2,24)=3.29, p=.06]$. A trend existed for participants to initiate a saccade more quickly in the go-to-either condition than in the go-to-old condition [383 msec vs. $435 \mathrm{msec}, t(12)=2.01, p=.07$ ]. La- tencies were not significantly different between the go-to-new condition and the go-to-either condition [393 msec vs. $383 \mathrm{msec}, t(12)=.49, p=$ .63]. Go-to-old latencies and go-to-new latencies were also significantly different $[t(12)=2.81, p<.01]$. This analysis was based on far fewer data points.

4. Although we have rejected a substantial portion of the trials, this is not unusual for experiments in which the collection of data pertinent to the question at hand depends upon the participants' behavior up to the point of and after the delivery of the measuring event (e.g., Klein \& MacInnes, 1999; MacInnes \& Klein, 2003). Rejection rates were approximately equal across conditions, with two notable exceptions. Stimulus placement restrictions led to a greater percentage of trials in which the probe could not be delivered in the nonpersistent $(30 \%)$ relative to persistent (14\%) and new item (15\%) probes. It is unlikely that this discrepancy affected saccadic reaction time in a meaningful way. Additionally, participants were less likely to saccade to the probe in the persistent and nonpersistent conditions ( $66 \%$ and $73 \%$, respectively) relative to the new probe condition ( $83 \%$ ), reflecting a strong bias to saccade to the new item even when the saccade target was explicitly indicated with the probe marker.

(Manuscript received July 17, 2003; revision accepted for publication October 28, 2003.) 